\title{
(Was) Kann Familienpolitik zur Gleichstellung der Geschlechter beitragen?
}

\author{
Silke Bothfeld \\ Christina Klenner
}

\begin{abstract}
Familienpolitik hat eine anhaltende Hochkonjunktur. Im Jahr 2001 wurde der Erziehungsurlaub reformiert und die Elternzeit eingeführt: Beide Elternteile konnten Elternzeit nun gleichzeitig und kombiniert mit Teilzeit in Anspruch nehmen, auch auf ein Jahr verkürzt mit höherem Elterngeld. 2004 wurde dann das Tagesbetreuungsausbaugesetz verabschiedet, das den Ausbau von Kinderbetreuungsplätzen für die unter Dreijährigen anstoßen sollte. Die neue Bundesfamilienministerin hat nachgelegt und an die Pläne der rot-grünen Koalition angeknüpft: Der Einführung des neuen einkommensabhängigen Elterngeldes 2007 folgt derzeit die Festschreibung eines Rechtsanspruchs auf einen Krippenplatz und dessen Umsetzung bis 2013 durch den Ausbau öffentlicher Einrichtungen oder Tagesmütter. Sogar die Wirtschaftsvertreter fordern den Ausbau der Kinderbetreuung und ergreifen immer öfter selbst die Initiative zur Einführung familienfreundlicher Maßnahmen in ihren Betrieben. Und an den lokalen Bündnissen für Familie beteiligen sich auch die
\end{abstract} DGB-Gewerkschaften, für die auf der Bundesebene Familienpolitik ebenfalls zu einem wichtigen Thema geworden ist (vgl. Stockfisch und Menne in diesem Heft). Wird mit diesen Aktivitäten nun ein Teil dessen eingelöst, was Frauenpolitikerinnen seit den 1970er Jahren fordern?

Wir denken, Skepsis ist angebracht, zumal die neue Familienpolitik zuvorderst im Dienste ökonomischer Ziele steht, namentlich die „Freisetzung" des weiblichen und die Förderung des kindlichen Humankapitals sowie die Rettung des Generationenvertrags beim umlagefinanzierten Rentensystem. Die Wirkungen sollten daher kritisch beobachtet werden. Als Prüfsteine für eine emanzipatorische Familienpolitik schlagen wir die soziale Absicherung von Erziehenden, die Erhöhung der Handlungsautonomie der Eltern und die Orientierung an einem egalitären Geschlechtermodell vor. Und schließlich wäre die Sorgearbeit als Gegenstand der Familienpolitik unzureichend definiert, wenn nur die Versorgung von Kindern, nicht aber von Älteren, gemeint wäre. Diese Anforderungen sind mit der ökonomischen Verwertungslogik wohl kaum in Einklang zu bringen.

Die soziale Sicherheit und die gerechte Behandlung von Erziehenden wird sich daran messen lassen müssen, inwiefern Erziehende unterschiedlich behandelt werden bzw. von institutionellen Lösungen in unterschiedlichem Maße profitieren, und generell, auf welche Weise sich Erwerbsunterbrechungen oder Arbeitszeitreduzierungen aufgrund von Elternschaft in der Fortsetzung beruflicher Karriere, auf Status und Geld auswirken. Momentan ist zu befürchten, dass die neue Familienpolitik zu neuen sozialen Strukturierungen führt, etwa wenn die Inanspruchnahme von Elterngeld, Elternurlaub oder Kinderbetreuung ungleich verteilt ist oder nicht für alle gleichermaßen zu einer sozialen Absicherung führt.

Die elterliche Handlungsautonomie wäre vor allem dann gegeben, wenn eine angemessen entlohnte und sichere Beschäftigung die Grundlage für die individuell selbstbestimmte Ausgestaltung des Privatlebens bildet. Dies gilt vor allem für die gering entlohnten und möglicherweise zudem prekär beschäftigten Erwerbstätigen in Dienstleistungsbereichen und anderswo. Zugespitzt formuliert: Familienpolitische Strategien sind kritisch zu werten, wenn sie nur auf Basis billiger und unsicherer Beschäftigung, z. B. in der häuslichen Pflege oder der Kinderbetreuung, realisiert werden können (vgl. Lutz und Theobald in diesem Heft).

Egalitäre Strategien zeichnen sich schließlich dadurch aus, dass institutionalisierte Angebote für Männer und Frauen gleichermaßen attraktive Lösungen darstellen und "Wahlfreiheit" eben nicht als ", Wahlpflicht der Mütter"verstanden wird. Dies gilt vor allem für erwerbstätige Mütter, deren Opferbereitschaft, d.h. Rückzug vom Arbeitsmarkt, nicht als Bedingung für die Realisierung von Vereinbarkeit stillschweigend in Kauf genommen werden darf. Vielmehr sollte die Arbeitsteilung von Frauen und Männern als ein Teil des Vereinbarkeitsproblems betrachtet und politisch behandelt werden (vgl. Jurczyk und Ellingsæter/ Leira).

Die neue Familienpolitik zielt teilweise auf die Veränderung dieser klassischen Arbeitsteilung, indem sie die Erwerbstätigkeit von Müttern und die Fürsorge von Vätern fördert. Doch ist die traditionelle Arbeitsteilung nur eine der Grundlagen, auf der die Ungleichheit der Geschlechter aufbaut, denn Frauen werden auch unabhängig von Fürsorgeaufgaben diskriminiert, etwa beim Einkommen oder beim beruflichen Aufstieg. Deshalb darf der Aufschwung in der Familienpolitik nicht dazu verleiten, gleichstellungspolitische Themen hintan zu stellen (vgl. Klenner).

Selbst wenn sich allmählich auch in Deutschland ein weniger traditionelles Modell herausbildet (vgl. Rüling), so ist die gleichstellungspolitische Abstinenz bedenklich. Die Gefahr wächst hinter dem Rücken der Politikerinnen und Politiker, die sich ganz der Familienpolitik zuwenden, nämlich dort, wo die soziale Sicherheit und die Chance auf eine Existenz sichernde Erwerbsarbeit als Grundlage für ein selbstbestimmtes Leben mit oder ohne Familie schwinden. Und dies erfordert sogar mehr als zusätzliche gleichstellungspolitische Aktivitäten: nämlich eine nach haltige Entwicklung des Sozialstaats, in dem die vielfältigen und wechselhaften Bedürfnisse der Menschen, und nicht eine eng definierte ökonomische Leistungsfähigkeit, den Bezugspunkt staatlichen Handelns bilden. Kinder zu bekommen und großzuziehen, ist nur einer dieser Wechselfälle.

\footnotetext{
Silke Bothfeld, Dr., Wissenschaftlerin im WSI in der Hans-BöcklerStiftung. Arbeitsschwerpunkte: Arbeitsmarktpolitik, Frauenerwerbstätigkeit, vergleichende Wohlfahrtsstaatsforschung. e-mail: silke-bothfeld@boeckler.de Christina Klenner, Dr., Wissenschaftlerin im WSI in der Hans-BöcklerStiftung. Arbeitsschwerpunkte: Frauenerwerbstätigkeit, Geschlechterverhältnisse, Work-Life Balance. e-mail: christina-klenner@boeckler.de
} 